\title{
Silencing of survivin by YM155 induces apoptosis and growth arrest in hepatocellular carcinoma cells
}

\author{
CHANGHE ZHANG, XIAOFEI CAO, YONGXIANG GEI, YONG WANG, \\ GUIYUAN LIU, GUOCHANG CHENG ${ }^{*}$ and QINGHONG LIU* \\ Department of General Surgery, Taizhou Peoples' Hospital, Taizhou, Jiangsu 225300, P.R. China
}

Received August 2, 2014; Accepted April 24, 2015

DOI: $10.3892 / \mathrm{ol} .2015 .3451$

\begin{abstract}
Survivin overactivation is a frequent event in human hepatocellular carcinoma (HCC), due to its function in the induction of hepatocyte proliferation and apoptotic dysfunction. Recently, a novel survivin inhibitor named YM155, has demonstrated broad antitumor effects against various malignant tumors. Therefore, the present study aimed to explore how this agent may impact on HCC and elucidate its underlying mechanism of action. Immunohistochemical analysis was performed on 8 specimens of human $\mathrm{HCC}$, to assess the protein expression of survivin and phosphorylated retinoblastoma tumor suppressor ( $\mathrm{p}-\mathrm{Rb}$ ). In addition, in vitro, HepG2 and Huh7 human HCC cell lines were exposed to $100 \mu \mathrm{M}$ YM155 for up to $72 \mathrm{~h}$ and the cell viability was subsequently determined using MTT assay. Furthermore, the apoptotic status of YM155-treated HCC cells was investigated by flow cytometry, and the protein levels of survivin, procaspase-3 and p-Rb in YM155-treated HCC cells were assessed by immunoblotting analysis. The results demonstrated that HCC specimens expressed high levels of survivin and $\mathrm{p}-\mathrm{Rb}$ protein compared with those of adjacent noncancerous liver tissues. In vitro, YM155 significantly induced HCC cell apoptosis and growth arrest. At the protein level, YM155 markedly inhibited survivin and p-Rb expression, and elevated procaspase-3. YM155 demonstrated significant antitumor effects on HCC cells in the present study. These effects were associated with its anti-proliferative and apoptosis-induction activities. YM155 requires further investigation as a novel agent for potential use as a therapeutic strategy for the treatment of HCC.
\end{abstract}

Correspondence to: Dr Qinghong Liu, Department of General Surgery, Taizhou Peoples' Hospital, 399 Hailing Southern Road, Taizhou, Jiangsu 225300, P.R. China

E-mail: docliuqinghong@163.com

*Contributed equally

Key words: survivin, YM155, hepatocellular carcinoma, apoptosis, proliferation, retinoblastoma tumor suppressor

\section{Introduction}

Hepatocellular carcinoma (HCC) is responsible for numerous clinical therapeutic problems and is ranked as the third leading cause of cancer-associated mortality worldwide (1). As only patients with early-stage HCC are able to achieve optimal survival by radical hepatectomy or liver transplantation, the majority of patients are beyond curative intervention upon HCC diagnosis $(1,2)$. Notably, existing alternative strategies, including chemotherapy and immunotherapy, have seldom elicited satisfactory responses in $\operatorname{HCC}(2,3)$. Therefore, the development of novel therapeutic agents against HCC is urgently required.

Survivin belongs to the family of apoptosis inhibitors (IAPs). Accumulating evidence has revealed that survivin overexpression is a critical contributor to HCC pathogenesis via its anti-apoptotic and pro-mitotic activities $(4,5)$. In addition, survivin possesses a distinct feature of high expression specificity in cancer, in contrast to that of normally differentiated tissues (6). Thereby, survivin represents an attractive therapeutic target for the development of antitumor agents, although progression in this field has remained static.

YM155 is a recently developed survivin inhibitor (6). To date, this novel, small, imidazolium-based chemical has exhibited broad antitumor effects on various tumors, including Wilm's tumor, glioblastoma and breast cancer (6-9). In addition, ongoing studies have demonstrated that YM155 may also enhance the chemosensitivity of cells to existing anticancer agents $(4,10,11)$. Furthermore, phase II clinical trials of YM155 for the treatment of non-small cell lung carcinoma are currently underway (7). However, the mechanisms underlying YM155 antitumor activity have not yet been fully elucidated, and the potent effect of YM155 in HCC remains unclear. Therefore, the present study aimed to investigate how and via what mechanism YM155 functions in HCC.

\section{Materials and methods}

Cells and reagents. Human HCC cell lines HepG2 and Huh7 were purchased from Shanghai Laboratory Animal Centre (SLRC; Shanghai, China). YM155 was obtained from Selleck Chemicals (Houston, TX, USA). Dulbecco's modified Eagle's medium (DMEM), fetal bovine serum (FBS) and MTT were purchased from Sigma-Aldrich (St. Louis, MO, USA). 
Annexin V/fluorescein isothiocyanate (FITC) apoptosis detection kit (cat. no. 556547) was purchased from BD Pharmingen (San Diego, CA, USA). Bicinchoninic Acid (BCA) Protein Assay and Protein Lysis kits, phosphate-buffered saline (PBS), streptomycin and penicillin were purchased from the Beyotime Institute of Biotechnology (Nanjing, China). The primary rabbit monoclonal anti-survivin (cat. no. 76424), anti-procaspase 3 (cat. no. 32499), anti- $\beta$-actin (cat. no. 8227) and anti-phosphorylated retinoblastoma tumor suppressor protein (p-Rb; Ser807/811; cat. no. D20B12) antibodies, as well as secondary goat anti-rabbit horseradish peroxidase-conjugated antibody (cat. no. 97080) were purchased from Abcam (Cambridge, MA, USA).

Immunohistochemistry. To explore the protein expression profiles of survivin and $\mathrm{p}-\mathrm{Rb}$, eight paraffin-embedded HCC specimens ( $\mathrm{T}$ group) and matched adjacent normal tissues (N group) were retrieved from the Department of Pathology (Taizhou People's Hospital, Taizhou, China) for immunohistochemical assessment. All patient samples recruited to the present study were approved by the ethical review committee (Institutional Ethical Board of Taizhou People's Hospital).

Consecutive sections ( $4 \mu \mathrm{m})$ of paraffin-embedded normal and tumor specimens were prepared as described previously (12). Survivin and p-Rb protein expression in these sections was detected by incubation with respective antibodies against survivin (1:150) and p-Rb (1:150) at $37^{\circ} \mathrm{C}$ for $1 \mathrm{~h}$. Two individual pathologists, blinded to patient characteristics, scored the sections using an Olympus CX32 microscope (Olympus Corp., Tokyo, Japan). Protein expression was classified according to the staining intensity as follows: 0 , absence of staining; 1, mild expression; 2, moderate expression and 3, high expression. A mean percentage of positive tumor cell was determined in at least five areas at $\mathrm{x} 400$ magnification. The percentage of positive tumor cells and the staining intensity were multiplied to produce a weighted score for each case. Theoretically, the total scores ranged from $0(0 \%$ of cells stained) to $3(100 \times 3 / 100)$.

Cell culture and viability measurement. Human HCC cell lines HepG2 and Huh7 were cultured in DMEM supplemented with $10 \%$ FBS and $1 \%$ streptomycin and penicillin, in an atmosphere of $5 \% \mathrm{CO}_{2}$ at $37^{\circ} \mathrm{C}$. Cells were allowed to adhere overnight. Subsequently, HCC cells incubated with and without YM155 were designated as the YM155 and control groups, respectively.

For cell viability assessment, HCC cells were seeded in 96-well plates (Nunc, Roskilde, Denmark) at 5000 cells/well and subsequently exposed to YM155 at the $\mathrm{IC}_{50}$ concentration of $100 \mathrm{nM}$ according to previous literature (13) for up to $72 \mathrm{~h}$. At various time-points $(0,24,48$ and $72 \mathrm{~h}), 20 \mu \mathrm{l}$ MTT $(5 \mathrm{mg} / \mathrm{ml})$ was added to each well. The HCC cells were then incubated at $37^{\circ} \mathrm{C}$ for a further $4 \mathrm{~h}$. The culture medium was removed and $150 \mu 10.1 \%$ dimethylsulfoxide (DMSO) was added to each well to resolve the MTT. The absorbance of each well was measured at $450 \mathrm{~nm}$ on a Multiskan FC 51119000 photometer (BioTek Instruments, Inc., Winooski, VT, USA). For apoptosis measurements and protein sample preparation, cells were cultured in 6-well plates (Nunc) with YM155 or without YM155 (control group) and harvested $48 \mathrm{~h}$ later.
Apoptosis detection. The apoptotic degree of YM155-treated HCC cells was quantified by flow cytometry using the FITC Annexin V Apoptosis Detection kit I. Briefly, cell samples were sequentially incubated for $15 \mathrm{~min}$ at $28^{\circ} \mathrm{C}$ with $5 \mathrm{mg} / \mathrm{ml}$ Annexin V-FITC (AV-FITC) and $10 \mathrm{mg} / \mathrm{ml}$ propidium iodide (PI) diluted in Annexin $\mathrm{V}$ binding buffer. The cells were then resuspended in PBS and analyzed with a flow cytometer (FACSCalibur; BD Biosciences, San Jose, CA, USA), using a 530/30 nm signal detector for AV-FITC and a 582/42 nm signal detector for PI. The data were subsequently analyzed by Flow J software (version 7.6.5; Tree Star, Inc., San Carlos, CA, USA). The upper left and lower left quadrants represented late and early apoptosis, respectively. The total apoptosis ratio was calculated by adding the late and early apoptosis proportions.

Western blotting. Cell samples grown in 6-well plates were incubated with ice-cold lysis buffer [0.1\% Triton X-100, $50 \mathrm{mM}$ HEPES (pH 7.5), 150 mM NaCl, 10\% (v/v) glycerol, $1.5 \mathrm{mM}$ $\mathrm{MgCl}_{2}, 1 \mathrm{mM}$ dithiothreitol, $1 \mathrm{mM}$ sodium fluoride, $0.1 \mathrm{mM}$ sodium orthovanadate, $1 \mathrm{mM}$ phenylmethylsulfonyl fluoride and $2 \mathrm{mg} / \mathrm{ml}$ leupeptin and aprotinin]. The total protein sols were centrifuged at $12,000 \mathrm{x} \mathrm{g}$ at $4^{\circ} \mathrm{C}$ for $10 \mathrm{~min}$ and the supernatants were bolted at $100^{\circ} \mathrm{C}$ by iron heating for a further $10 \mathrm{~min}$. A BCA Protein Assay kit was used to determine the protein concentration. Equivalent samples (30 mg protein) were then subjected to $12 \%$ SDS-PAGE (Beyotime Institute of Biotechnology). The proteins were transferred to nitrocellulose membranes (Beyotime Institute of Biotechnology) and probed with specific primary antibodies. The antibodies were used at the following concentrations: survivin $(1: 1,500)$, procaspase-3 $(1: 2,000), \mathrm{p}-\mathrm{Rb}$ $(1: 2,000), \beta$-actin $(1: 2,000)$, followed by secondary antibody $(1: 2,500)$. The molecular sizes of the target proteins were determined by comparison with prestained Bio-Rad protein markers (Bio-Rad Laboratories, Inc., Hercules, CA, USA). The protein bands were then scanned using the Western Lightning Chemiluminescent Reagent Plus (Perkin Elmer, Boston, MA, USA) detection system. Protein immunoreactivity was quantified by densitometry analysis using the Gel DocXR System 170-8170 device and (Bio-Rad Laboratories, Inc., Nazareth, Belgium) and Quantity One software (version 4.4; Bio-Rad Laboratories, Inc.). $\beta$-actin was used as a loading control.

Statistical analysis. All parametric data are expressed as the mean \pm standard error of the mean, and analyzed by Student's t-test, whereas non-parametric data analysis was performed by Mann-Whitney U test. For all tests, analyses were performed using SPSS 19.0 statistical software (IBM SPSS, Armonk, NY, USA) and two-sided $\mathrm{P}<0.05$ was considered to indicate a statistically significant difference.

\section{Results}

Survivin and $p-R b$ protein expression is enhanced in $H C C$ cells. To investigate the variance in protein expression of survivin between human HCC and normal liver tissues, immunohistochemistry assays were performed. As shown in Fig. 1, HCC specimens presented with markedly greater survivin expression than that of noncancerous tissues, and the distribution was characterized by expression primarily in the nucleus, but also the cytoplasm. 
A

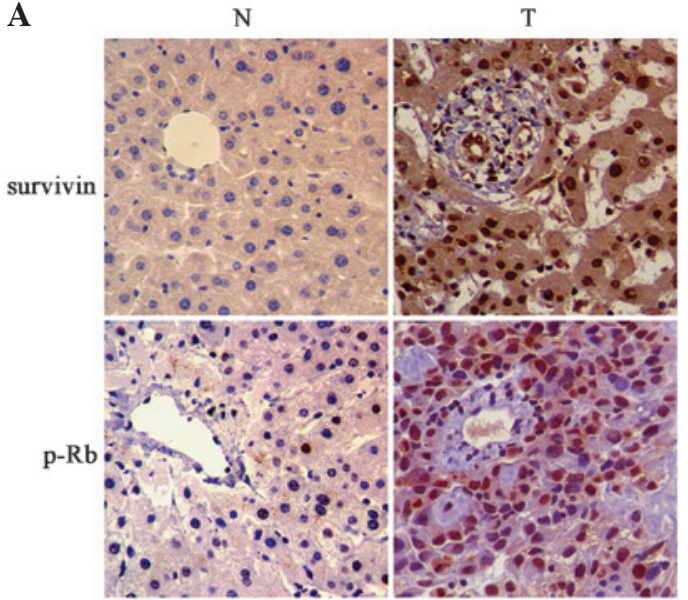

B

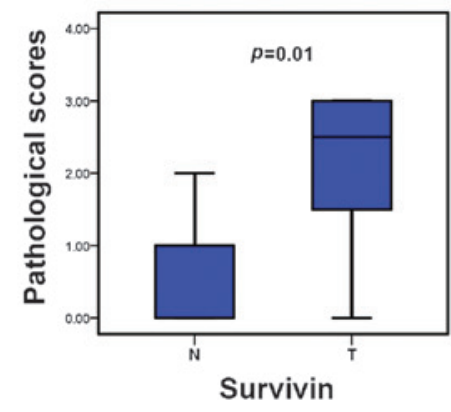

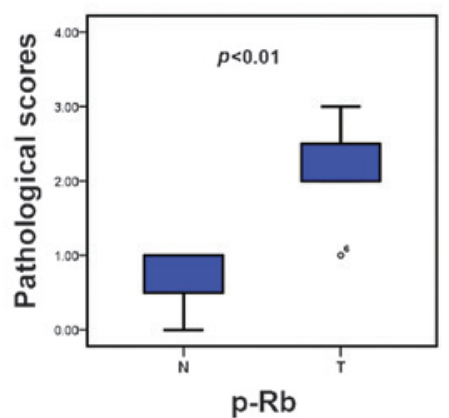

Figure 1. Protein expression of survivin and $\mathrm{p}-\mathrm{Rb}$ in $\mathrm{HCC}$ and adjacent normal tissues. (A) Immunohistochemical analysis revealed that the HCC specimens exhibited evident nucleic protein expression of survivin and $\mathrm{p}-\mathrm{Rb}$, compared with that of adjacent noncancerous liver tissues (magnification, $\mathrm{x} 400$ ). (B) Quantification of survivin and $\mathrm{p}-\mathrm{Rb}$ expression revealed enhanced expression in HCC tissues. ( $\mathrm{n}=3, \mathrm{P}<0.01)$. N, normal tissue; T, HCC tissue; $\mathrm{p}-\mathrm{Rb}$, phosphorylated retinoblastoma tumor suppressor; HCC, hepatocellular carcinoma.
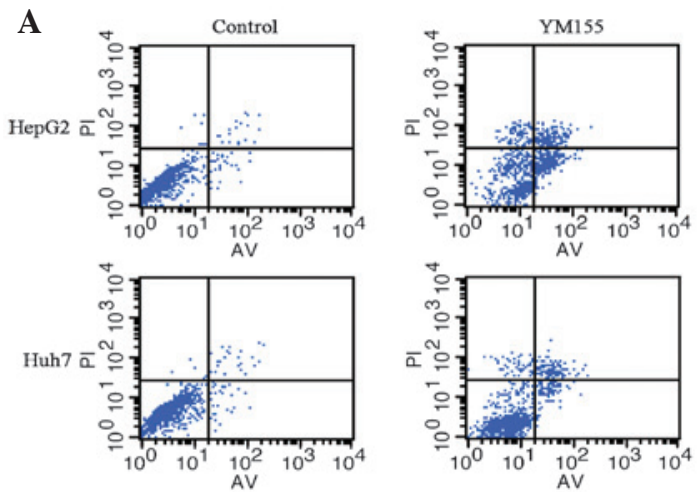

D

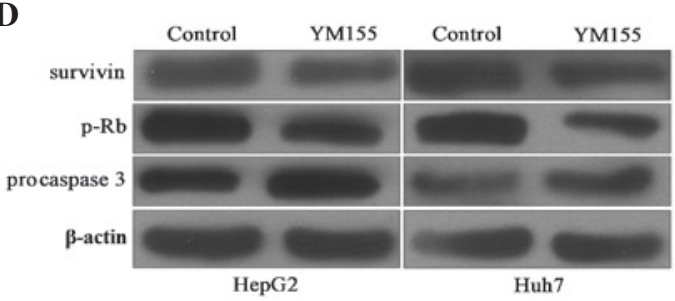

B

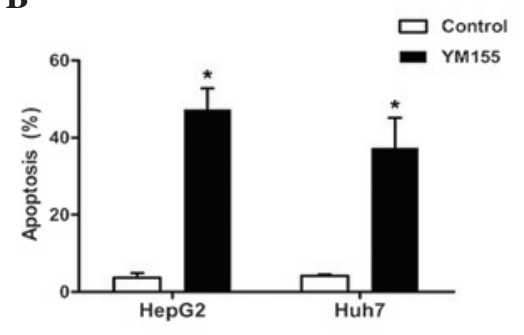

C

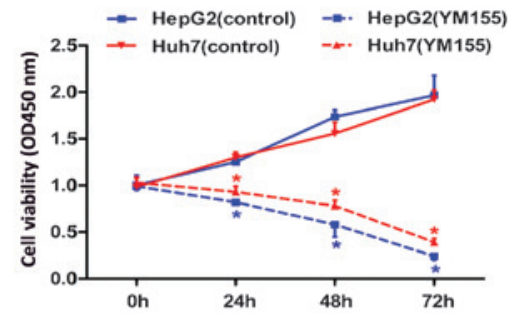

$\mathbf{E}$

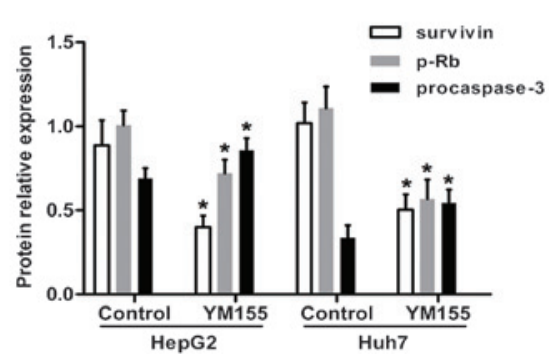

Figure 2. YM155 induces cell apoptosis and proliferation arrest in HCC cell lines. (A and B) The apoptosis ratios of HepG2 and Huh7 cells were significantly elevated by YM155 treatment. (C) In MTT cell viability assays, YM155 notably suppressed the cell proliferation of cancerous hepatocytes. (D and E) YM155 significantly inhibited survivin and $\mathrm{p}$ - $\mathrm{Rb}$ protein expression, and promoted procaspase- 3 expression as determined by western blotting and densitometry (relative to $\beta$-actin). ( $\mathrm{n}=3,{ }^{*} \mathrm{P}<0.05$ ). HCC, hepatocellular carcinoma; $\mathrm{p}$-Rb, phosphorylated retinoblastoma tumor suppressor; PI, propidium iodide; AV, Annexin V.

It is well established that $\mathrm{Rb}$ protein functions as a cell-cycle suppressor, and its functional loss through protein phosphorylation has been implicated to the tumorigenesis of various types of neoplasm $(14,15)$. Therefore, the phosphorylation level of $\mathrm{Rb}$ protein in HCC was investigated. Immunohistochemical analysis revealed that HCC samples displayed elevated $\mathrm{p}-\mathrm{Rb}$ expression, as compared with that of normal liver tissues, in a nucleus-exclusive distribution model (Fig. 1).

YM155 promotes HCC cell apoptosis. Cell apoptosis status was determined by flow cytometry. Following $48 \mathrm{~h}$ of exposure to YM155, the cell apoptosis ratios in HepG2 and Huh7 cells were $47.3 \pm 5.5$ and $37.3 \pm 7.8 \%$, respectively, markedly higher than those of the corresponding control groups, which were $3.7 \pm 1.2$ and $4.2 \pm 0.4 \%$, respectively $(\mathrm{P}<0.05$; Fig. $2 \mathrm{~A}$ and $\mathrm{B})$. These results indicated a significant pro-apoptotic effect of YM155 on HCC cell lines.

YM155 induces growth arrest in HCC cell lines. To verify the antitumor activity of YM155, HCC cell viabilities were investigated by MTT assay. Following YM155 exposure, HepG2 cell viabilities at 24,48 and $72 \mathrm{~h}$ were $0.81 \pm 0.02,0.55 \pm 0.09$ and $0.25 \pm 0.05$ respectively, which were markedly lower than those at the corresponding times in the control group $(1.00 \pm 0.04$, $1.74 \pm 0.10$ and $1.97 \pm 0.19)(\mathrm{P}<0.05$; Fig. 2C). Concurrently, the 
Huh7 cell survival at 24, 48 and $72 \mathrm{~h}$ in the YM155 group was $0.93 \pm 0.06,0.78 \pm 0.06$ and $0.38 \pm 0.06$, respectively. These results were markedly lower than those at the corresponding time-points in the control group: $1.30 \pm 0.06,1.56 \pm 0.12$ and $1.92 \pm 0.10$ ( $\mathrm{P}<0.05$; Fig. 2C). These data demonstrated a significant anti-proliferative effect of YM155 in HCC cells.

YM155 inhibits survivin and $p$-Rb protein expression and enhances procaspase-3. To detect the variations in the protein expression of survivin, $\mathrm{p}-\mathrm{Rb}$ and procaspase-3 in HCC cells following YM155 pretreatment, immunoblotting assays were performed (Fig. 2D and E). The relative survivin protein expression levels of YM155-treated HepG2 and Huh7 cells were $0.40 \pm 0.07$ and $0.50 \pm 0.09$, respectively; these were markedly lower than those of the corresponding control groups $(0.89 \pm 0.15$ and $1.02 \pm 0.12$, respectively). Relative $\mathrm{p}-\mathrm{Rb}$ protein levels in HepG2 and Huh7 cells following YM155 exposure $(0.72 \pm 0.08$ and $0.57 \pm 0.12$, respectively) were also lower than those in the associated control group $(1.01 \pm 0.09$ and $1.11 \pm 0.13$, respectively). The relative protein expression levels of procas-

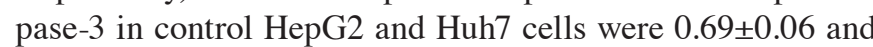
$0.34 \pm 0.08$, respectively, which were significantly lower than those of the corresponding YM155-treated groups $(0.86 \pm 0.07$ and $0.53 \pm 0.08$, respectively). These results revealed that YM155-treatment was not only able to directly suppress survivin protein expression, but also indirectly inhibited $\mathrm{p}-\mathrm{Rb}$ and enhanced procaspase- 3 expression.

\section{Discussion}

In the present study, the protein expression of survivin and $\mathrm{p}-\mathrm{Rb}$ in HCC tissues was evaluated, and the apoptosis and proliferation status of YM155-treated HCC cells was examined. The results demonstrated that silencing of survivin expression, via specific inhibitor YM155, induced HCC cell apoptosis and growth arrest. These results corroborated those of previous studies regarding other neoplasms $(8-11,13,16)$. In order to represent a potential therapeutic strategy for $\mathrm{HCC}$, the impacts of survivin, as well as YM155, on HCC should be sufficiently understood and validated.

Apoptosis is a natural defense mechanism for the elimination of unhealthy cells, and the loss of sensitivity to apoptosis has been identified as a hallmark of cancer (6,17-19). Accumulating evidence has revealed that survivin overactivation contributes to apoptotic dysfunction via the intrinsic and extrinsic apoptosis pathways (4,7,17-20). Given that survivin contains a single BIR domain, similarly to other IAP family members, it is not surprising that survivin is able to interfere with the activity of caspases in the mediation of apoptosis (18-20). In addition, survivin has demonstrated the ability to prevent the release of apoptosis-induced factor from the mitochondrial intermembrane space, protecting cells against apoptotic death $(19,21,22)$. Futhermore, survivin also suppresses apoptosis via inhibition of the pathway of death receptors and downstream tumor necrosis factor-related apoptosis-inducing ligand $(13,18,23,24)$. The present study was limited to examination of the total apoptosis profile and the protein expression levels of procaspase-3, a principle 'executor', committing cells to death via the intrinsic apoptosis pathway $(19,25)$. The results of the present study revealed that YM155 positively modulated the intrinsic apoptosis pathway and enhanced the total apoptosis ratios in HCC cells, which was consistent with the results of previous studies (18-20).

Notably, the survivin distribution identified by immunohistochemical analysis exhibited evident characteristics of expression in the nucleus first and cytoplasm second. These findings were in accordance with an early hypothesis that survivin is able to shuttle between the nucleus and cytoplasm through active nucleocytoplasmic transport $(6,19)$. On the other hand, the presence of cytoplasmic survivin has been further associated with poor prognosis in HCC, as members of the caspase protein family occur predominantly in the cytoplasm $(5,6,19)$. Combining these factors with another generally accepted notion that survivin expression may correlate negatively with the distal prognosis of cancer patients even following radical resection, indicates that postoperative immunohistochemistry may be of interest in the selection of postoperative therapeutic schedules for patients with HCC, through assessment of survivin expression levels and distribution $(5,6,18)$.

Apart from its role in apoptosis suppression, survivin represents a widely recognized mitotic regulator of various cell division processes $(6,18-20)$. Notably, survivin is able to participate in the chromosomal passenger complex, and thereby accelerate chromosomal segregation and cytokinesis $(18,19)$. In addition, survivin is also able to promote the formation of microtubules and the spindle. Furthermore, the DNA damage-mediated activation of checkpoint kinase 2 always results in secondary survivin release and apoptosis dysfunction, facilitating the survival of tumorous cells $(18,20)$. To date, accumulating evidence has exemplified the proliferative impact of survivin on various tumor cells $(6,8,18-19)$. In the current study, the results of cell viability analysis supported this hypothesis. However, the potential anti-proliferative and pro-apoptotic effects of YM155 were indistinguishable by MTT assessment, and therefore the phosphorylation level of $\mathrm{Rb}$ protein was evaluated. $\mathrm{Rb}$ is another generally acknowledged cell-cycle regulator, downstream of survivin $(20,26,27)$. Rb protein primarily presents as a hypophosphorylated form within quiescent cells, whereas its phosphorylation promotes cell proliferation and tumorigenesis $(14,15,20,26-28)$. In the present study, $\mathrm{p}-\mathrm{Rb}$ was used as an indicator to assess the proliferation profile of HCC cells. The results revealed that YM155 induced the phosphorylation of Rb protein, which indicated a proliferative effect of survivin on HCC cell lines.

Survivin is a significant therapeutic target for the development of anticancer agents, due to its role in tumorigenesis and exclusive expression in tumor cells $(18,19)$. However, targeting survivin may be difficult as it is not an enzyme or a cell surface protein (7,13). Recently, low molecular weight YM155 has emerged as a novel breakthrough drug, associated with existing survivin inhibitors. In addition to direct potential antitumor activity, YM155 has also displayed an ability to sensitize HCC cells to other chemotherapeutic agents $(10,11,18-20)$.

Cancer is essentially a consequence of an imbalance between cell death and proliferation $(18,19)$. Aberrant cell proliferation, combined with apoptotic resistance, is a crucial contributor to tumorigenesis (18-20). In this context, YM155 may possess potential to be a dual 'protector' for patients with 
HCC, by suppressing survivin. The results of the present study preliminarily exhibited the potency of YM155 on HCC cells via blocking cell growth and enhancing apoptosis. Further studies are required in order to elucidate whether this novel agent may be utilized as a potential therapeutic strategy for HCC.

\section{References}

1. Guglielmi A, Ruzzenente A, Conci S, et al: Hepatocellular carcinoma: Surgical perspectives beyond the barcelona clinic liver cancer recommendations. World J Gastroenterol 20 : 7525-7533, 2014

2. Zhang Y, Shi ZL, Yang X and Yin ZF: Targeting of circulating hepatocellular carcinoma cells to prevent postoperative recurrence and metastasis. World J Gastroenterol 20 : 142-147, 2014.

3. Shindoh J, Kaseb A and Vauthey JN: Surgical strategy for liver cancers in the era of effective chemotherapy. Liver Cancer 2 47-54, 2013.

4. Zhao X, Ogunwobi OO and Liu C: Survivin inhibition is critical for Bcl-2 inhibitor-induced apoptosis in hepatocellular carcinoma cells. PLoS One 6: e21980, 2011.

5. Liu JL, Zhang XJ, Zhang Z, Zhang AH, Wang W and Dong JH: Meta-analysis: Prognostic value of survivin in patients with hepatocellular carcinoma. PLoS One 8: e83350, 2013.

6. Cheung CH, Huang CC, Tsai FY, et al: Survivin - biology and potential as a therapeutic target in oncology. Onco Targets Ther 6: 1453-1462, 2013.

7. Yamanaka K, Nakata M, Kaneko N, et al: YM155, a selective survivin suppressant, inhibits tumor spread and prolongs survival in a spontaneous metastatic model of human triple negative breast cancer. Int J Oncol 39: 569-575, 2011.

8. Tao YF, Lu J, Du XJ, et al: Survivin selective inhibitor YM155 induce apoptosis in SK-NEP-1 Wilms tumor cells. BMC Cancer 12: 619, 2012.

9. Lai PC, Chen SH, Yang SH, Cheng CC, Chiu TH and Huang YT: Novel survivin inhibitor YM155 elicits cytotoxicity in glioblastoma cell lines with normal or deficiency DNA-dependent protein kinase activity. Pediatr Neonatol 53: 199-204, 2012.

10. Liang H, Zhang L, Xu R and Ju XL: Silencing of survivin using YM155 induces apoptosis and chemosensitization in neuroblastomas cells. Eur Rev Med Pharmacol Sci 17: 2909-2915, 2013.

11. Koike H, Nitta T, Sekine Y, et al: YM155 reverses rapamycin resistance in renal cancer by decreasing survivin. J Cancer Res Clin Oncol 140: 1705-1713, 2014.

12. Wang H, Jiang S, Zhang Y, et al: High expression of thymosin beta 10 predicts poor prognosis for hepatocellular carcinoma after hepatectomy. World J Surg Oncol 12: 226, 2014.
13. Charette N, De Saeger C, Horsmans Y, Leclercq I and Stärkel P: Salirasib sensitizes hepatocarcinoma cells to TRAIL-induced apoptosis through DR5 and survivin-dependent mechanisms. Cell Death Dis 4: e471, 2013.

14. Reed CA, Mayhew CN, McClendon AK, Yang X, Witkiewicz A and Knudsen ES: RB has a critical role in mediating the in vivo checkpoint response, mitigating secondary DNA damage and suppressing liver tumorigenesis initiated by aflatoxin B1. Oncogene 28: 4434-4443, 2009.

15. Nojiri S and Joh T: Albumin suppresses human hepatocellular carcinoma proliferation and the cell cycle. Int J Mol Sci 15: 5163-5174, 2014.

16. Kaneko N, Mitsuoka K, Amino N, et al: Combination of YM155, a survivin suppressant, with bendamustine and rituximab: A new combination therapy to treat relapsed/refractory diffuse large B-cell lymphoma. Clin Cancer Res 20: 1814-1822, 2014.

17. Fabregat I: Dysregulation of apoptosis in hepatocellular carcinoma cells. World J Gastroenterol 15: 513-520, 2009.

18. Mobahat M, Narendran A and Riabowol K: Survivin as a preferential target for cancer therapy. Int J Mol Sci 15: 2494-2516, 2014.

19. Coumar MS, Tsai FY, Kanwar JR, Sarvagalla S and Cheung CH: Treat cancers by targeting survivin: Just a dream or future reality? Cancer Treat Rev 39: 802-811, 2013.

20. Rauch A, Hennig D, Schäfer C, et al: Survivin and YM155: How faithful is the liaison? Biochim Biophys Acta 1845: 202-220 2014

21. Zeng J, Sun Y, Wu K, et al: Chemopreventive and chemotherapeutic effects of intravesical silibinin against bladder cancer by acting on mitochondria. Mol Cancer Ther 10: 104-116, 2011.

22. Zhang R, Humphreys I, Sahu RP, Shi Y and Srivastava SK: In vitro and in vivo induction of apoptosis by capsaicin in pancreatic cancer cells is mediated through ROS generation and mitochondrial death pathway. Apoptosis 13: 1465-1478, 2008.

23. Taniguchi H, Horinaka M, Yoshida T, et al: Targeting the glyoxalase pathway enhances TRAIL efficacy in cancer cells by downregulating the expression of antiapoptotic molecules. Mol Cancer Ther 11: 2294-2300, 2012.

24. Sakai T, Eskander RN, Guo Y, et al: Flavokawain B, a kava chalcone, induces apoptosis in synovial sarcoma cell lines. J Orthop Res 30: 1045-1050, 2012.

25. Loor G, Kondapalli J, Iwase H, et al: Mitochondrial oxidant stress triggers cell death in simulated ischemia-reperfusion. Biochim Biophys Acta 1813: 1382-1394, 2011.

26. Baker SJ and Reddy EP: CDK4: A key player in the cell cycle, development and cancer. Genes Cancer 3: 658-669, 2012.

27. $\mathrm{Hu} \mathrm{H}, \mathrm{Li} \mathrm{Z}$, Chen J, et al: P16 reactivation induces anoikis and exhibits anti tumour potency by down regulating Akt/survivin signalling in hepatocellular carcinoma cells. Gut 60: 710-721,2011.

28. Deng L, Lu Y, Zhao X, et al: Ran GTPase protein promotes human pancreatic cancer proliferation by deregulating the expression of Survivin and cell cycle proteins. Biochem Biophys Res Commun 440: 322-329, 2013. 